\title{
OP-32 ANALYSING INEQUALITY IN USE OF HEALTHCARE SERVICES: IMPLICATIONS FOR TARGETING WITHIN UNIVERSAL HEALTH COVERAGE REFORMS
}

Shankar Prinja, Pankaj Bahuguna, Deepak Balasubramaniam, Atul Sharma, Rajesh Kumar. School of Public Health, Post Graduate Institute of Medical Education and Research, Chandigarh (Haryana), India

\subsection{6/bmjgh-2016-EPHPabstracts.32}

Background Equity is at the heart of any attempt to universalize health care. However, given the limited resources available for health in India, 'progressive universalism' appears to be more realistic than comprehensive universal health coverage (UHC) for the entire population. This calls for some form of targeting, either based on wealth status, geography, caste or any other factor. The choice of means for targeting will depend on two factors: (1) the extent of inequality across each of the population groups; and (2) the ease of detecting the worse offs using a given criteria. In this paper, we analyse data from a household survey from Haryana state, to understand which inequalities in utilisation of health care services are important. This will help orient policy for best targeting the public subsidies.

Methods Multi-stage systematic random sampling was used to select 51,656 households across 21 districts of Haryana state, India. Coverage of preventive (maternal and child health services) as well as curative (outpatient care and hospitalisation) services was assessed, along with evaluation of financial risk protection. Coverage of maternal and child health services was studied based on eight indicators: seven indicators concerning 
maternal and child health and one indicator concerning family planning. Inter-district inequalities were compared based on four indicators: full antenatal care, full immunisation, institutional delivery and contraceptive prevalence rate. Intra-district inequalities were analysed by evaluating the coverage of three indicators at sub-centre level: full antenatal care, full immunisation and institutional delivery.

Similarly, for each of the indicators for preventive and curative service coverage and financial risk protection we assessed wealth status, caste, education and occupation. Wealth-based inequalities were assessed using a concentration index. A summary index of UHC was computed which was also assessed across each district. Geometric mean was used to aggregate individual indicators and compute the summary index.

Findings Population coverage for preventive and curative services is presented. Adjusting for wealth-based inequality, the coverage for all the indicators was less than the unadjusted coverage by $0.1 \%$ to $6.7 \%$ in absolute terms and by $0.1 \%$ to $27 \%$ in relative terms. Coverage of services was lowest among the disadvantaged groups including those belonging to Muslim community, Scheduled Caste, Scheduled Tribes, and those without literacy and/or formal education. There was no significant effect of occupation. The summary index for UHC varied from $71 \%$ in Kurukshetra district to $12 \%$ in Mewat district. Significantly high inter- and intra-district inequalities were observed.

Conclusion Geographic inequalities are more important than wealth-based inequalities in Haryana state. There is need to re-orient targeting strategies, applying a geographic approach, while moving towards universal health care.

Grant funding (National Rural Health Mission, Haryana) for research but no other competing interests. 